\title{
MODELO DE COORDINACIÓN DE INVENTARIOS COLABORATIVOS SOPORTADO EN UN ENFOQUE SOCIOTÉCNICO DE SISTEMAS
}

\section{Collaborative inventory coordination model supported in a sociotechnical approach of systems}

\author{
Oscar Palacio-León ${ }^{1}$, Marianna Barrios-León ${ }^{2}$, Wilson Adarme Jaimes ${ }^{3}$ \\ ${ }^{1}$ M.Sc. Universidad Militar Nueva Granada (Bogotá D.C., Colombia). oscar.palacio@unimilitar.edu.co \\ ${ }^{2}$ Dra. Ing. Universidad de Carabobo (Valencia, Venezuela).marianna.barrios@gmail.com \\ ${ }^{3}$ Ph.D. Universidad Nacional de Colombia (Bogotá D.C., Colombia). wadarmej@unal.edu.co
}

(Recibido octubre 23 de 2017 y aceptado noviembre el 15 de 2017)

\begin{abstract}
Resumen
Este trabajo presenta un modelo alternativo de coordinación de inventarios para la integración vertical entre un proveedor un almacén con múltiples clientes y una familia de productos. La originalidad del modelo estriba en tres elementos principales. La primera es que el nivel de servicio ofrecido sigue un enfoque deductivo, utilizando a priori un marco matemático definido. La segunda es que la política de gestión de la disponibilidad de la unidad de carga eficiente está administrada por el proveedor. La tercera es que el diseño metodológico emplea un validador técnico-económico. En primer lugar, se realiza una revisión de la literatura para posicionar la investigación. A continuación, se presentan los principales elementos metodológicos usados, principalmente el procedimiento para valorar el impacto del enfoque sociotécnico de sistemas y perfeccionamiento del modelo AIU en que soporta el esquema de colaboración. Finalmente, los resultados de validación del modelo se presentan y discuten en forma crítica.
\end{abstract}

Palabras clave: Empresa extendida; empresa saludable; mecanismo de coordinación; inventarios colaborativos; modelo Administración-Imprevistos-Utilidad.

\begin{abstract}
This paper presents an alternative model of inventory coordination for vertical integration between a supplier and multiple customers. The originality of the model lies in three main elements. The first is that the level of service offered follows a deductive approach, using a priori a mathematical definite model. The second one is that the supplier directs the strategy of management of the availability of the collaborative inventory. The third one is that the methodological design uses a technical - economic validator. First, the literature checked to position the investigation. Later, they present the principal methodological elements that were used, principally the procedure to value the impact of the approach sociotécnico of systems load and improvement of the model ARU in that supports the scheme of collaboration. Finally, the validation results of the model are presented and analyzed in critical form.
\end{abstract}

Keywords: Extended Company; healthy company; coordination mechanism; collaborative inventories; model Administration-Relief-Utility.

\section{INTRODUCCIÓN}

La triada estado-universidad-empresa en la última década ha venido acuñando el concepto empresa saludable, con el propósito de hacer de la promoción y prevención de la salud laboral una política de sostenibilidad [9], enfocada hacia la mejora continua de la productividad, calidad, entre otros aspectos, a través de la implementación de un enfoque sociotécnico de sistemas [8], impulsado por el Agente Principal de la Red de Suministros [10], que como visión estratégica del negocio da origen al termino empresa extendida [3], esquema empresarial que permite crear valor compartido [16]. Es decir, hablar 
de empresa saludable (Ergónomos) es equivalente a empresa extendida (Logísticos), desde la perspectiva del enfoque sociotécnico de sistemas.

El problema de Diseño de la Empresa Extendida Dirigida por la Demanda (EEDD) se puede resolver por diferentes enfoques. El que se propone en el presente trabajo tiene su origen en la Gestión de Inventarios Colaborativos. Más aun, [8] consideran que este enfoque sinergia la creación de valor compartido entre los agentes, cuando la integración de la EEDD se apoya en la mejora de las condiciones físicas, psicológicas, sociales y organizacionales que protegen y promueven la seguridad y la salud de la fuerza laboral. De hecho, este paradigma se aplica a los entornos colaborativos, en los que hay una normatividad consensuada que orienta los lineamientos de la EEDD, en procura de unos fines $u$ objetivos comunes de productividad soportados en inversiones en valor de vida [15].

En este sentido, [5] sostienen que intervenir en forma integral los factores de riesgo psicosociales de la fuerza laboral, le implicara al Agente Principal de la EEDD hacer inversiones en valor de vida, que se verán reflejadas con mejoras entre un 60 a un $90 \%$ e incluso más en los niveles de productividad. Así mismo, [10] establecieron que un mecanismo de coordinación orientado a la toma de decisiones conjunta, lo constituye la economía de movimientos con enfoque hacia la mejora de la productividad saludable, que permite ofrecer niveles de servicio por encima del $85 \%$, con respecto a la gestión del inventario de la EEDD. De igual manera, [9] determino que al intervenir el área de operaciones a través de un enfoque sociotécnico de sistemas, los tiempos de respuesta intraempresa se ven mejorados por encima del $15 \%$ e incluso más, cuando el criterio decisional se fundamenta en el estudio de la productividad saludable, conducente a la mitigación de la sensación de desgaste laboral.

Entre tanto, [8] lograron establecer que invertir en valor de vida 12 dólares por colaborador-año, le permitiría obtener ingresos operacionales cercanos a los 23 dólares por colaborador-año, a través del diseño de una política sostenible de gestión de inventarios colaborativos, soportado en un mecanismo de coordinación embebido en criterios de productividad saludable. Por ende, el presente artículo tiene como propósito proponer un modelo de coordinación de inventarios colaborativos con enfoque sociotécnico de sistemas, que sea gestionado por el agente principal de una EEDD, que está integrada por un proveedor un almacén central que suple una familia de productos a múltiples administradores de cuenta clave (KAM), a la vez, que concatena factores psicosociales con las inversiones requeridas en valor de vida, al tiempo que crea valor compartido, y asegura condiciones técnicoeconómicas atractivas para su negociación entre los interesados.

El documento se organiza como sigue. En la Sección 2, Método, se describe la metodología de integración y encadenamientos logísticos para la creación de valor compartido, que soporta el modelo de inventarios colaborativo propuesto. En la Sección 3, Resultados, se encontró que la puesta en marcha de la política de gestión del inventario conjunto diseñada, para los interesados en la integración de la EEDD Líder del sector de las autopartes de la ciudad de Bogotá D.C, satisface los requerimientos exigidos por el sponsor de la misma, en atención al Modelo AdministraciónImprevistos-Utilidad (AIU), exigencias que se indican en estricto orden de aparición, como: 4,5\%-5\%-10\%. En la Sección 4, Análisis, se presentan los principios, relaciones y generalizaciones que arrojan los resultados obtenidos de la investigación, en contraste con el objetivo propuesto. Finalmente, en la Sección 5 , Conclusión, se exponen los análisis con referencia al modelo de integración propuesto para la EEDD estudiada, que soporta la política de gestión del inventario colaborativo diseñada bajo un enfoque sociotécnico de sistemas.

\section{MÉTODO}

La metodología en que se apoya el modelo de coordinación de inventarios colaborativos con enfoque sociotécnico de sistemas, consta de tres etapas a saber: i) Análisis sociotécnico de sistemas de la EEDD; ii) Formulación del modelo de inventarios colaborativos; y iii) Evaluación técnico-económica del proceso de integración de la EEDD.

\subsection{Análisis sociotécnico de sistemas de la EEDD}

En esta primera fase, se hace una intervención a las condiciones intralaborales que influyen en la salud y bienestar de la fuerza laboral, para lo cual, se empelo el modelo demanda-control-apoyo social [2], el cual, se armoniza con el modelo de desequilibrio-esfuerzorecompensa [2], los cuales a su vez son articulados por medio del modelo dinámico de factores de riesgo 
psicosociales [15]. Con el propósito de establecer, que características del entorno de trabajo y de la organización, son significativas para ser mejoradas mediante la ejecución de inversiones en valor de vida, representadas en: i) El montaje del Sistema Inmótico de la EEDD; y ii) Los Costos de Operación y Posesión de Programas de Promoción, Prevención y vigilancia del absentismo y bienestar laboral al interior de la EEDD [8-10].

Por otra parte [9], formulo el Índice Global de Productividad Saludable (IGPs), en función de la data obtenida de la operatividad del modelo dinámico de factores de riesgo psicosociales, la cual se transforma en unidades de capacidad de sistema con respecto a la salud: i) Física (Condiciones materiales); ii) Mental (Contenido del trabajo); y iii) Social (Organización del trabajo). Según, este autor el IGps mide el desempeño alcanzado por la fuerza laboral, a partir de la intervención y mejora de la percepción en la fuerza laboral, con respecto a la cultura ergonómica que posee la EEDD [12]. Es así que, el IGps deberá estar por encima del $85 \%$, para que sea considerada la EEDD de clase mundial, porque emplea buenas prácticas empresariales con soporte en principios de colaboración y de buen gobierno [9], encaminadas a la mitigación del absentismo, mejora del bienestar de la fuerza laboral y a la creación de valor compartido, como consecuencia del equilibrado entre las inversiones en valor de vida, y las estrategias orientadas a la continuidad del negocio [8]. Este índice está conformado por los tres ratios que se recogen en la ecuación 1.

$$
I G_{P S}=\prod_{i=1}^{3}\left[\left(R_{C T}\right) *\left(R_{C M}\right) *\left(R_{O T}\right)\right] * 100
$$

En donde: i) $R_{C T}=$ Contenido del Trabajo (Una evaluación de este ratio, por debajo del $100 \%$ refleja que el sistema productivo pérdidas de velocidad con relación al recurso humano por fatiga u por restricciones inevitables en la secuenciación de la producción); ii) $R_{C M}=$ Condiciones Materiales (Un ratio de Condiciones materiales menor al 100\%, indica que las instalaciones industriales presenta situaciones inseguras para con el trabajador principalmente, con respecto a la fisiología del trabajo, en especial atención a la seguridad industrial y al confort del sitio de trabajo); y iii) Ror = Organización del Trabajo (Un ratio de organización del trabajo inferior al 100\%, exterioriza que el sistema productivo posee pérdidas de calidad de vida, asociadas con situaciones de convivencia entre operarios u por condiciones a típicas en la subordinación laboral).

\subsection{Formulación del modelo de inventarios colaborativos}

En la segunda fase, se diseñó el modelo de inventario colaborativo multieslabón [6], soportado en un mecanismo de coordinación operacional [1], para la toma de decisiones conjunta en tamaño de lote homogéneo multiproducto, gestionada por el Agente Principal (AP) de la EEDD. Es decir, el AP decide qué tamaño de lote conservar en Inventario de Seguridad en la EEDD, y a su vez, es garante de la administración del volumen en Inventario de Consumo a distribuir durante el cicló económico, en atención a las limitaciones en: i) Inversiones en valor de vida; ii) Capacidad de bodegaje del Centro de Distribución (CEDIS); iii) Presupuesto en inventario de Seguridad; y iv) Ajuste por desviaciones en Inventario de Consumo. A continuación, se expone su modelo matemático:

$$
\begin{aligned}
& D_{t}=C_{t}+S_{t}+K_{O} \\
& U_{t}=\rho Y_{t-1}, \text { con } 0<\rho<1 \\
& U_{t}-U_{t-1}=\rho\left(Y_{t-1}-Y_{t-2}\right) \\
& \quad S_{t}=(1+\mu)\left[\rho\left(Y_{t-1}-Y_{t-2}\right)\right], \text { con } \mu>0 \\
& E_{C}(L D)=a t^{2}+b t+c \\
& K_{O}=C_{W}+I_{V v}=E_{C}(L D) \\
& E_{C}(L I)=D_{t}-(2+\mu) \rho D_{t-1}+(1-\mu) \rho D_{t-2} \\
& E_{C}(L I)=E_{C}(L D), \text { con } t \geq 2
\end{aligned}
$$

En donde: i) $D t=$ Ingreso Operacional Total en el periodo $t$; ii) $\mathrm{Ct}=$ Inventario de Consumo Vendido en el Periodo $t$; iii) St = Inventario de Seguridad en el Periodo $t$; iv) Ko = Capital de Trabajo Autónomo Base; v) $\rho=$ Consumo Per-Capital, que se espera sea mantenido de un periodo a otro, por parte de los clientes en favor de la EEDD; vi) $\mu=$ Mecanismo de Aceleración del Volumen del Inventario de Seguridad pactado entre los agentes involucrados; vii) $\mathrm{CW}=$ Capital de trabajo base; y viii) IVV = Inversiones en valor de vida (Sistema Inmótico mas Bienestar de la fuerza laboral y sus familias).

Al operativizar por métodos numéricos la ecuación 9, más exactamente, a través del uso de método de búsqueda directa de la solución completa, para la Ecuación en Diferencia Finita de tipo Lineal de Segundo Orden con Forma Polinómica de grado $n$ [4]. Ecuación balanceada que se constituye por sí sola, en un mecanismo de coordinación del inventario monoagente basado en los principios económicos de [7], al que se ha denominado: Modelo de Expectativas y Ciclos de Existencias Conjuntas Sostenible. Otro rasgo de este mecanismo de coordinación, es que simula las variaciones cíclicas del inventario conjunto, y a su vez, permite que estas sean amortizadas en el largo plazo 
de la consolidación de la EEDD, tal y como, se expresa en la ecuación 10.

$D_{\mathrm{t}, \mathrm{GC}}=\mathrm{D}_{\mathrm{t}, \mathrm{GH}}+\mathrm{D}_{\mathrm{t}, \mathrm{PC}}$

$D_{\mathrm{t}, \mathrm{GH}}=\left(\mathrm{C}_{1}\right)\left(\mathrm{r}_{1}\right)^{\mathrm{t}}+\left(\mathrm{C}_{2}\right)\left(\mathrm{r}_{2}\right)^{\mathrm{t}}$

$\mathrm{D}_{\mathrm{t}, \mathrm{PC}}=\frac{a}{3} \mathrm{t}^{2}+\left(\frac{3 b-2 a}{9}\right) \mathrm{t}+\left(\frac{9 c-3 b-a}{27}\right)$

En donde: i) $D_{t, G H}=$ Solución General Homogénea; ii) $D_{t, P C}=$ Solución Particular Completa Constante; $y$ iii) $D_{t, G C}=$ Solución General Completa Sostenible.

El resultado obtenido a través de la ecuación 10 , se incorporan en la función de costo total que gestiona el inventario conjunto de la EEDD, por parte del AP [9], la cual se presenta en la ecuación 13.

$$
\mathrm{CT}_{\text {Mínimo }}(\mathrm{T})=\mathrm{D}_{\mathrm{t}, \mathrm{GC}}+\frac{H+n C}{T}+\frac{T * i * D_{t, G C}}{T}
$$

En donde: i) $n=$ Número total de items por orden conjunta; ii) $\mathrm{H}=$ Costo Conjunto de Ordenar; iii) $\mathrm{C}=$ Costo de Ordenar Asociado a cada KAM; iv) $T$ = Intervalo de Tiempo entre Pedidos por Año; y v) $i=$ Tasa de Transferencia Anual Administra por el Agente Principal.

Realizando la operatividad clásica a la ecuación 13 , se obtiene el modelo de inventario colaborativo para la EEDD intervenida, que se sustenta en la formulación matemática que se indica a continuación [10]:

$$
\begin{aligned}
& \mathrm{T}^{*}=\sqrt{\frac{2 *(H+n C)}{i * D_{t, G C}}} \\
& S K U_{i j}^{10}=\left[\left(\frac{\left.\left.D_{t, G C^{*}\left(T^{*}+L_{j}\right)}\right) * W_{j}\right]}{52 * P v_{i}}\right)\right] \\
& U C E_{i j}^{10}=\left[\left[S K U_{i j}^{10} * \rho\right]\right\rceil \\
& I S_{i}^{10}=\left[\left[S K U_{i j}^{10} *(1-\rho)\right]\right] \\
& \mathrm{CT}\left(T^{*}\right)=\left(1+\mathrm{i} * T^{*}\right) * \mathrm{D}_{\mathrm{t}, \mathrm{GC}} \\
& \mathrm{F}_{\mathrm{A}}=\frac{U C E_{A P}^{10}}{U C E_{A N}^{10}} \\
& \mathrm{~F}_{\mathrm{B}}=\frac{U C E_{A N}^{10}}{U C E_{A P}^{10}} \\
& C T_{U C E}^{E E D D}=\operatorname{Min}\left\{\frac{1}{2}\left[F_{A}+F_{B}\right]\right\} * \operatorname{CT}\left(T^{*}\right)
\end{aligned}
$$

En donde: i) $T^{*}=$ Intervalo de Tiempo Óptimo entre Pedidos para Múltiples Ítems; ii) $S K U_{i j}=$ Unidad básica de bodegaje en base diez por ítem i y por agente negociador $j$; iii) $L_{j}=L$ Lead time negociado con cada agente $j$; iv) La suma de $\left(T^{*}+L\right)$ debe ser un entero y estar dado en semanas; $v) I S_{i}=$ Inventario de Seguridad por Ítem i; vi) $P v_{i}=$ Precio de Venta Unitario del Ítem i; vii) $W_{j}=$ Participación en el Mercado del item i; viii) $U C E_{i j}=$ Unidad de Carga Eficiente en base diez por item i y por agente negociador j; ix) $C T\left(T^{*}\right)=$ Costo Total de Gestión de la UCE por parte del agente principal; $y x)$ CTUCE${ }_{E E D D}=$ Costo total de la estrategia de suministros colaborativa para la EEDD.
2.3 Evaluación técnico-económica del proceso de integración de la EEDD

En esta última fase, se recurre al planteamiento de escenarios para el inventario colaborativo propuesto, los cuales son evaluados en términos financieros, es decir, mediante la construcción de los Flujos de Caja Totalmente Neto, que asocian tanto los aspectos técnicos como la gestión del riesgo, con la tasa de oportunidad compuesta deseada por el AP de la EEDD [8]. Esta evaluación se soporta en criterios de toma de decisiones financieras en proyectos, tales como: i) VPN $_{\text {Ajustado; }}$ y ii TIRModificada; entre otros criterios de evaluación, a fin de establecer la viabilidad económica de la mejor estrategia de negociación del inventario conjunto, con el cual se configura la EEDD que involucre no solo los aspectos cuantitativos sino también a los cualitativos, al robustecerla con un análisis de sensibilidad a los parámetros de la función de costo total del inventario conjunto.

\section{RESULTADOS}

Para mostrar la importancia del trabajo expuesto, fue usado un caso de estudio. Se tomó como referente una compañía líder del sector de autopartes de Colombia con sede en la ciudad de Bogotá, para lo cual, se siguieron los pasos que se describen a continuación:

\subsection{Cuantificar el IGPs para el periodo de planificación} de la UCE, mediante un análisis sociotécnico de sistemas para la EEDD en estudio

A partir de los resultados obtenidos, por la implementación de la Batería de Instrumentos, para la Evaluación de Factores de Riesgo Psicosocial. En especial, la Data relacionada con las condiciones intralaborales, que en la actualidad la fuerza laboral percibe que son causantes del diestrés que está presente en la EEDD intervenida [2]. Por medio de la cual, se obtienen los parámetros que se indican a continuación: i) Contenido del Trabajo $=6.3 \%$; ii) Condiciones Materiales $=8.1 \%$; y iii) Organización del Trabajo $=3.9 \%$.

Por otro lado [9], determino un factor de ajuste al índice de riesgo del ítem ii) de 1.07, derivado de la evaluación del diseño espacial del área de operativa, es decir, la ideal vs la real que posee el AP de la EEDD. Continuando con el análisis, se procedió a transformar estos parámetros en los factores de tiempo, que se citan en seguida: i) Tiempo Efectivo de Producción del 
Personal $=93.7 \%$; ii) Tiempo Operativo del Personal $=$ 86.1\%; y iii) Tiempo Productivo Alcanzado por el Personal $=81.7 \%$. Factores que se relacionan entre sí, a través, de la ecuación 1 . En consecuencia, el IGps se cifra en un $82 \%$ aproximadamente, tal y como se muestra en la ecuación 22.

$$
\mathrm{IGPS}_{\mathrm{PS}}=\left(\frac{93.7}{100}\right)\left(\frac{86.1}{93.7}\right)\left(\frac{81.7}{86.1}\right) * 100 \approx 82 \%
$$

De la ecuación 22, se puede inferir que la mejora que puede alcanzar la EEDD posee un delta máximo del $18 \%$, con respecto al nivel de productividad actual. Delta máximo, que no iría en detrimento de la salud laboral, ya que no sobrepasa el índice de saturación deseado por el AP de la EEDD del 85\% [9].

\subsection{Determinar el mejor modelo de inventario} colaborativo para la EEDD en estudio

Comenzando por la proyección del inventario productivo total [8], a través de la utilización de las ecuaciones 2 a la 10. Que al ser operativizadas con sus respectivos parámetros [4], proyectan la expectativa económica durante el primer año de integración de la EEDD, es decir, para el año 2017. Prospectiva que se indican con la ecuación 23, y que corresponde, al uso de la Capacidad Disponible mínima de sostenibilidad empresarial para la familia de productos en análisis, la cual, se pretende que pacten los agentes interesados en la integración horizontal de la EEDD, dentro de la configuración de la estrategia, como tal, de gestión del inventario conjunto.

$\mathrm{D}_{17, \mathrm{GC}}=\mathrm{D}_{17, \mathrm{GH}}+\mathrm{D}_{17, \mathrm{PC}} \approx 1,667,220 \frac{U S D}{A \tilde{\mathrm{N}} O}$
Los parámetros suministrados por el $A P$ en esta primera parte del modelado, son los que se citan a continuación: i) $\rho=85 \%$ (Palacio-León, 2017); ii) $\mu=1.0235$ (Prospectiva de crecimiento del mercado meta de la EEDD); iii) $10_{2015}=$ $1,111,875$ USD/Año; iv) $1 O_{2016}=1,090,160$ USD/Año; v) $\alpha=$ 65\% (Capacidad Disponible mínima de sostenibilidad empresarial para la familia de productos en análisis); vi) $C_{W}$ $=437,000$ USD/Año; vii) $I_{V V}$ (Fija) = 43,000 USD/Año (Costo de administración del Sistema de Bienestar de la EEDD); $y$ viii) $I_{V V}$ (Variable) $=10 \mathrm{USD} / \mathrm{mes}$-Colaborador (Nómina conformada por 60 empleados).

Tomando como punto de partida la ecuación 23, se procede a cuantificar la UCE para el AP, CEDIS y KAMs, siguiendo estas pautas (Véase tabla 1):

i) UCEAP. Fue cuantificada mediante la aplicación de las ecuaciones 12 a la 17; ii) UCECEDIs. Se determinó mediante la utilización del Modelo EOIESJIT [11]; y iii)

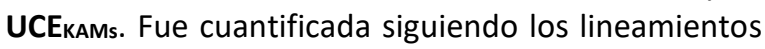
para la gestión de existencias al interior de la EEDD de [6]. Pasó seguido, se procedió a cubicar el volumen de UCE para la integración de la EEDD intervenida, para lo cual se empleó la ecuación 21 en forma exhaustiva, con el objeto de establecer los parámetros de coordinación del inventario conjunto a convenir, partiendo de: i) Los resultados agregados de los requerimientos en inventario de cada agente (Véase tabla 1); y ii) La meta desea por el AP en sobrecosto para la gestión del inventario conjunto. Lográndose establecer que el volumen base de Inbox requeridos por la EEDD es de 2,040 por año, el cual no genera un sobrecostos para el AP, proceso de simulación discreta que se presenta en forma completa para la UCE en la tabla 2.

Tabla 1. Configuración de la UCE para el primer año

\begin{tabular}{llllll}
\hline KAM & Lead Time (Semanas) & $\begin{array}{l}\text { Demanda Anual } \\
\text { (\%) }\end{array}$ & $\begin{array}{l}\text { Requerimiento en Inventario } \\
\text { (Primer Año de Integración) } \\
\text { UCE } \\
\text { (Inbox) }\end{array}$ & UCE $_{\text {CEDIs (Inbox) }}$ & UCE $_{\text {KAMs } \text { (Inbox) }}$ \\
\hline Zona Occidente & 1 & 25 & 440 & 420 & 310 \\
Zona Oriente & 1 & 15 & 270 & 240 & 180 \\
Zona Sur & 2 & 10 & 270 & 240 & 270 \\
Zona Norte & 2 & 20 & 530 & 460 & 520 \\
Institucional & 1 & 30 & 530 & 460 & 520 \\
\hline
\end{tabular}

Los parámetros suministrados por el AP, para esta parte del modelado son los que se citan a continuación: i) Se cuenta con cinco KAMs, sistema que le cuesta a la compañía en promedio el 35\% anual sobre Inbox; ii) SKU homogénea con un peso máximo de 25 kilos/Inbox; iii) Cubicaje que representa un ingreso neto operacional promedio de 70 USD/Inbox; iv) $H=150$ USD/Orden; v) $C=50$ USD/Orden-
KAM; vi) $i=30 \%$ anual; vii) Los costos de operación por Inbox corresponden al $40 \%$ de los ingresos que genera; viii) $L a$ Políticas de bodegaje es Caótica; ix) El costo de oportunidad del capital fue valorado a una tasa del $5 \%$ efectivo anual; $y$ x) El sobrecosto máximo admisible para la gestión del inventario colaborativo es del $10 \%$ anual. 
Tabla 2. Factibilidad de la UCE para el primer año

\begin{tabular}{lllll}
\hline \multirow{2}{*}{ CRITERIO TECNICO } & \multicolumn{2}{l}{ ESTRATEGIAS POTENCIALES DE INVENTARIOS COLABORATIVOS } \\
& ESCENARIO 1 & ESCENARIO 2 & ESCENARIO 3 & ESCENARIO 4 \\
\hline Sobrecosto (\%) & 9.6 & 6.6 & 4.8 & 0.0 \\
UCE (Inbox/Pedido) & 1,320 & 1,420 & 1,500 & 2,040 \\
\hline
\end{tabular}

3.3 Evaluación técnico-económica del proceso de integración de la EEDD

La tabla 3, recoge los resultados de la evaluación financiera, para cada una de las UCE que conforman el portafolio de estrategias en inventario colaborativo, que puede ser adoptada por la EEDD, las cuales se originaron a partir del proceso de simulación presentado en la tabla 2. Es de mencionar, que la evaluación técnico-económica fue realizada con una tasa de interés compuesta de oportunidad del $17.5 \%$ efectiva anual.
En términos generales, el AP de la EEDD Líder del sector de autopartes de la ciudad de Bogotá D.C, lo constituye la gerencia de operaciones, quien tiene el poder de negociación de la UCE en atención a: i) Es el agente que restringe la capacidad de efectiva de producción de la EEDD a 1,760 Inbox/Corrida de Producción; ii) Pacto para el primer año de la integración un inventario de consumo de 1,320 Inbox/Pedido, complementado con un inventario de seguridad de 440 Inbox/Pedido; y iii) Fijo el precio del servicio de bodegaje a los KAMs a un nivel de racionalización de 0,28 UDS/Posición y de 0,31 UDS/Inbox.

Tabla 3. Portafolio Técnico-Financiero para la UCE*

\begin{tabular}{lllll}
\hline CARACTERÍSTICAS DEL RRC PARA LA EEDD & \multicolumn{4}{c}{ Estrategias potenciales de Inventarios Colaborativos } \\
& ESCENARIO 1 & ESCENARIO 2 & ESCENARIO 3 & ESCENARIO 4 \\
\hline Capacidad de Bodegaje (Inbox) & 1,760 & 1,900 & 2,000 & 2,720 \\
Presupuesto de Inversiones (USD) & $\$ 1,137,200$ & $\$ 1,141,200$ & $\$ 1,176,200$ & $\$ 1,328,200$ \\
BANA (USD) & $\$ 691,944$ & $\$ 385,181$ & $\mathbf{\$ 3 3 , 5 3 8}$ & $\mathbf{( \$ 4 0 5 , 9 2 6 )}$ \\
TVR (EFECTIVO ANUAL) & $\mathbf{7 5 \%}$ & $\mathbf{5 0 \%}$ & $\mathbf{2 1 \%}$ & $\mathbf{( 3 0 \% )}$ \\
DECISIÓN & VIABLE & VIABLE & DEFERIR & ABANDONAR \\
\hline
\end{tabular}

\section{ANÁLISIS}

En términos generales, la colaboración se enfoca en una relación de ganar-ganar entre los agentes logísticos que la desarrollan [13]. Hecha esta salvedad, se puede inferir con base en los trabajos de [2] [8-10] y [12], además de la experiencia adquirida en el presente trabajo, que diseñar una estrategia de coordinación de inventarios colaborativos soportado en un enfoque sociotécnico de sistemas, fortalece las condiciones operativas del sistema productivo, al mejorar la percepción de la fuerza laboral y sus familias, con respecto al bienestar y mitigación del absentismo, más aún, como tema de estudio posee un gran potencial, tanto para la realización de investigaciones teóricas como para la implementación de aplicaciones prácticas.

De igual manera, incorporar la inversión en valor de vida al modelado del inventario colaborativo, con el objeto de integrar la EEDD bajo la arista de una arquitectura empresarial saludable [12], parte de la buena voluntad de los interesados en mejorar la calidad de vida laboral de la fuerza laboral y sus familias, que como estrategia de colaboración deberá ir mas halla del cumplimiento de la normatividad vigente en la materia [2], y mucho menos, soportar dicha decisión en forma radical en el efecto positivo que trae, con respecto a la creación valor compartido [16]. 
En lo que atañe, con el modelo prospectivo empleado para evaluar la inversión de vida con la gestión del inventario conjunto, se logró evidenciar que los econométricos se comportan bastante bien [8,9], con una data agregada entre uno a tres años inclusive, y ahí en adelante asumiendo dicha inversión constante, para el horizonte de planificación de la integración de la EEDD. Sin embargo, en esta investigación se logró demostrar que la capacidad de bodegaje se racionaliza aún más (Una reducción cercana al 65\%), con respecto a lo argumentado por los autores anteriormente citados, cuando la inversión en valor de vida se proyecta en forma polinómica con una tasa de crecimiento del $85 \%$, siendo el dato más relevante el Beneficio Anual Neto Ajustado que se vio mejorado en un $62 \%$ aproximadamente, cuando el diseñador de la política de inventarios colaborativos soportado en un enfoque sociotécnico de sistemas, contempla en el modelado el concepto microeconómico Ceteris paribus.

\section{CONCLUSIONES}

Incorporar el enfoque sociotécnico de sistemas al modelamiento del inventario colaborativo, hace del mecanismo de coordinación un elemento de equidad, entre el inventario de seguridad y el inventario para el consumo que brinda el AP a la EEDD, que a su vez, le permite brindar y garantizar al canal de ventas que gestiona este agente un nivel de servicio por encima del $85 \%$, al tiempo que crea valor compartido del $12 \%$ anual e incluso más (Tasa de descuento social anual válida para Colombia), para los interesados en la integración. Más aun, invertir una unidad económica en aspectos sociotécnicos de sistemas, representa que la alternativa de inventarios colaborativos generará riqueza a los interesados. Por ende, la alternativa genera riqueza con seguridad traerá consigo un beneficio social para la EEDD, que para el caso en estudio invertir un USD/Colaborador le representa un beneficio de 0.69 USD/Colaborador, además de recuperar dicha inversión.

Para finalizar, el AIU refleja los costos indirectos de la integración de la EEDD que está dispuesto a asumir el $A P$, con el objeto de garantizar la implementación del esquema de coordinación del inventario conjunto sostenible. Por otro lado, el porcentaje del AIU nunca es menor al $10 \%$ del valor total de la inversión requerida para la integración de la EEDD, y es un rubro variable según la complejidad de la alternativa de inversión, el cual es un indicador indispensable para conocer la aversión al riesgo y la seguridad que tiene el AP de ejecutar e iniciar la política de inventarios colaborativa diseñada. En suma, se encontró que la puesta en marcha de la política de gestión del inventario conjunto, valuada para los interesados en la integración de la EEDD líder del sector de las autopartes de la ciudad de Bogotá D.C, posee un riesgo moderado del $10.49 \%$ para el AP.

\section{REFERENCIAS}

[1] M. Albrecht, Supply chain coordination mechanisms: New approaches for collaborative planning. Berlin-Heidelberg, Germany: Springer Science \& Business Media, 2010, pp. 35-62.

[2] M. Barrios-León, "Efectos del desgaste laboral, como riesgo psicosocial, en la productividad", [Tesis Doctoral], Dpto. Ing. Industrial, Ing., UC, Valencia (Venezuela), 2014.

[3] E.W. Davis and R.E. Spekman, The Extended Enterprise: Gaining Competitive advantage through collaborative supply chains. New York, USA: FT Press, 2004, pp. 3-28.

[4] C. González y J.A. Barrios, Análisis discreto en Economía y Empresa. Madrid, España: Editorial AC, 2000, pp. 76-134.

[5] H.W. Hendrick \& B.M. Kleiner, Macroergonomics: Theory, Methods and Applications. Mahwah, New Jersey, USA: Lawrence Erlbaum Associates, Inc., Publishers, 2002, pp. 25-96.

[6] K. Kempf et al., Planning production and inventories in the extended enterprise: a state of the art handbook (Vol. 1). New York, USA: Springer Science \& Business Media, 2011, pp. 233-268.

[7] L.A. Metzler, (1941, Aug.). The nature and stability of inventory cycles. The Review of Economics and Statistics, 23(3), 113-129.

DOI: http://www.jstor.org/stable/1927555.

[8] O. Palacio-León, M. Barrios-León, y W. AdarmeJaimes, "Modelo de coordinación de inventarios colaborativos soportado en criterios de productividad saludable", IV-CIIO. Medellín, 2017, 10p.

[9] O. Palacio-León, “Equilibrado y diseño de líneas de ensamble mixtas orientados por criterios de productividad saludable", working paper, Facultad de Ingeniería, Programa de Ingeniería Industrial, Universidad Militar Nueva Granada-Sede Bogotá, 2017, 20p.

[10] O. Palacio-León \& W. Adarme-Jaimes, La higiene industrial como elemento de diseño de centros de distribución de mercancías urbanas. Cuadernos de 
Ingeniería: Innovación y tecnología de vanguardia, Vol. 1, 2015, pp. 118-129.

[11] O. Palacio-León, "Propuesta metodológica para el diseño y operación de instalaciones de almacenamiento modulares ecoeficientes para productos no perecederos", [Tesis Maestría], Dpto. Ing. de Sistemas e Industrial, Facultad de Ingeniería, Universidad Nacional de Colombia, Bogotá D.C (Colombia), 2012.

[12] T.L. Smith-Jackson, M.L. Resnick, \& K.T. Johnson, Cultural Ergonomics: Theory, methods, and applications. Boca Raton, USA: CRC Press, 2014, pp. 187-206.

[13] R.J. Trent, Gerencia estratégica de suministros: diseñe su propia fuente de ventaja competitiva.
Traductor María Teresa Aparicio, Bogotá, Colombia: Panamericana Editorial, 2018, pp. 120-160.

[14] G. Villalobos, A.M. Vargas, J. Escobar, M.L. Jiménez, \& M.A. Rondón, Batería de instrumentos para la evaluación de factores de riesgo psicosocial. Bogotá D.C, Colombia, Ministerio de la Protección Social, 2010, 99p.

[15] W.K. Viscusi, (2004, January). The value of life: estimates with risks by occupation and industry. Economic Inquiry, 42(1), 29-48.

DOI: https://doi.org/10.1093/ei/cbh042.

[16] J. Wieland, Creating Shared Value-Concepts, Experience, Criticism. Friedrichshafen-Württemberg, Germany: Springer, 2017, pp. 6-77. 